TITLE:

\title{
Dopant profiling on semiconducting sample by scanning capacitance force microscopy
}

\author{
$\operatorname{AUTHOR}(\mathrm{S}):$
}

Kobayashi, K; Yamada, H; Matsushige, K

\section{CITATION:}

Kobayashi, K ... [et al]. Dopant profiling on semiconducting sample by scanning capacitance force microscopy. APPLIED PHYSICS LETTERS 2002, 81(14): 2629-2631

\section{ISSUE DATE:}

2002-09-30

URL:

http://hdl.handle.net/2433/39652

\section{RIGHT:}

Copyright 2002 American Institute of Physics. This article may be downloaded for personal use only. Any other use requires prior permission of the author and the American Institute of Physics. 


\title{
Dopant profiling on semiconducting sample by scanning capacitance force microscopy
}

\author{
Kei Kobayashi ${ }^{\text {a) }}$ \\ International Innovation Center, Kyoto University, Kyoto 606-8501, Japan \\ Hirofumi Yamada and Kazumi Matsushige \\ Department of Electronic Science and Engineering, Kyoto University, Kyoto 606-8501, Japan
}

(Received 21 June 2002; accepted 6 August 2002)

\begin{abstract}
Scanning capacitance force microscopy (SCFM) capable of mapping differential capacitance $(\partial C / \partial V)$ on semiconducting sample based on atomic force microscopy (AFM) without an external capacitance sensor is introduced. While an electric field alternating at an angular frequency $\omega$ is applied between the tip and the sample, an induced electrostatic force (ESF) oscillating at its third harmonic frequency $(3 \omega)$ is detected by a lock-in amplifier. Owing to the fact that the magnitude of the induced ESF is proportional to the square of the magnitude of the applied electric field and the fact that the capacitance of the semiconducting sample is also modulated at $\omega$, the amplitude and the phase of the induced ESF oscillating at $3 \omega$ contain information on $\partial C / \partial V$. We present $\partial C / \partial V$ images on a Si test sample obtained by SCFM using both contact-mode AFM and dynamic-mode AFM, showing clear contrasts depending on species and density of dopants. (C) 2002 American Institute of Physics. [DOI: 10.1063/1.1510582]
\end{abstract}

Since the size of individual transistors in large-scale integrated circuits is becoming smaller and smaller, further advance in silicon technology requires quantitative threedimensional dopant profiling techniques with high spatial resolution. Owing to an inherent high spatial resolution of scanning probe microscopy (SPM), scanning capacitance microscopy (SCM) is becoming one of the promising dopant profiling tools. ${ }^{1}$

The first $\mathrm{SCM}^{2}$ was implemented using an insulating stylus with an electrode and an uhf resonance capacitance sensor. $^{3}$ The stylus was scanned on a sample surface by contact. Shortly the uhf resonance capacitance sensor was combined with scanning tunneling microscope ${ }^{4}$ and atomic force microscope (AFM). ${ }^{5}$ Since then, SCM based on AFM with a resonance capacitance sensor has become very common SPM which can map capacitance variation. Since there is a parasitic capacitance between a probe tip and a sample surface that is much larger than that just beneath the tip, SCM is usually used to map differential capacitance $(\partial C / \partial V)$ on a semiconducting sample utilizing a lock-in detection technique. This technique is based on the fact that capacitance of a semiconducting sample beneath the probe tip can be modulated by applying an alternating electric field. The lateral and capacitance resolution of SCM is limited by the tip-sample contact area and the sensitivity of the capacitance sensor, respectively. In other words, reducing the contact area for improvement of the lateral resolution will deteriorate the signal-to-noise ratio of the capacitance sensor output.

On the other hand, it was demonstrated about ten years ago that the capacitance derivative $(\partial C / \partial z)$ can be measured by detecting an electrostatic force (ESF) induced by an applied electric field. ${ }^{6,7}$ The basic principle was as follows. A bias voltage $V_{t}$ is applied to a cantilever tip while a sample is

${ }^{a)}$ Electronic mail: keicoba@iic.kyoto-u.ac.jp electrically grounded. An induced attractive $\mathrm{ESF}, F$, is expressed as

$$
F=\frac{1}{2} \frac{\partial C}{\partial z} V_{t}^{2}
$$

where $z$ is the axis normal to the sample surface. Assuming that $V_{t}$ is composed of dc bias voltage $V_{\mathrm{dc}}$ and ac bias modulation voltage $V_{\mathrm{ac}}$ alternating at an angular frequency $\omega\left(V_{t}\right.$ $\left.=V_{\mathrm{dc}}+V_{\mathrm{ac}} \cos \omega t\right), F$ could be written as

$$
F=\frac{1}{4} \frac{\partial C}{\partial z}\left(V_{\mathrm{ac}}^{2}+V_{\mathrm{ac}}^{2} \cos 2 \omega t\right)
$$

supposing $V_{\mathrm{dc}}=0$ for convenience. Thus $\partial C / \partial z$ component could be measured by detecting $2 \omega$ component by a lock-in amplifier (LIA). This method has not been common probably because the topographic artifacts cannot be eliminated.

Based on this method, we developed a scanning capacitance force microscopy (SCFM) that can map $\partial C / \partial V$ on semiconducting samples instead of $\partial C / \partial z$. The basic idea of our SCFM is to detect an induced ESF oscillating at the third harmonic frequency $(3 \omega)$. On a semiconducting sample, $\partial C / \partial z$ is not constant but is dependent of applied bias voltage. Thus the function $\partial C(V, z) / \partial z$ can be approximated to be composed of a dc component and an ac component when we apply $V_{t}$. Therefore we suppose the following equation:

$$
\frac{\partial C(V, z)}{\partial z} \simeq \frac{\partial C\left(V_{\mathrm{dc}}, z\right)}{\partial z}+\frac{\partial^{2} C\left(V_{\mathrm{dc}}, z\right)}{\partial V \partial z} V_{\mathrm{ac}} \cos \omega t .
$$

Accordingly the induced ESF would contain $3 \omega$ component $\left(F_{3 \omega}\right)$ which can be expressed as

$$
F_{3 \omega} \simeq \frac{1}{8} \frac{\partial^{2} C\left(V_{\mathrm{dc}}, z\right)}{\partial V \partial z} V_{\mathrm{ac}}^{3} \cos 3 \omega t .
$$

Since the differential capacitance $\partial^{2} C\left(V_{\mathrm{dc}}, z\right) / \partial V \partial z$ contains information on the variation of the differential capacitance 


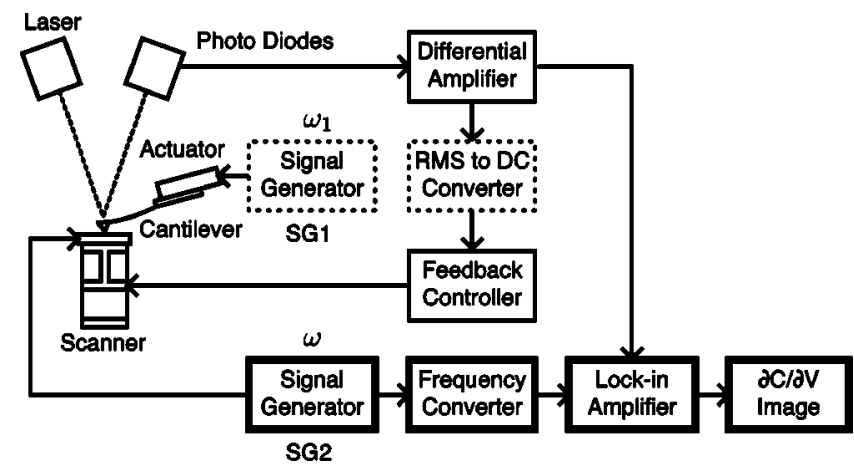

FIG. 1. Schematic diagram of experimental setup for SCFM using contactmode AFM and dynamic-mode AFM. The components required for operating dynamic-mode AFM are indicated as dotted rectangles. The extra components required for mapping $\partial C / \partial V$ are signal generator, frequency converter, and lock-in amplifier, which are indicated as thick rectangles.

$(\partial C / \partial V)$ on the sample surface, two-dimensional mapping of $\partial C / \partial V$ might be possible by detecting $3 \omega$ component ESF.

In Fig. 1, a schematic diagram of the experimental setup for SCFM both in contact-mode AFM and in dynamic-mode AFM is shown. All the experiments described in this letter were carried out under ambient conditions. The cantilevers used were highly-doped Si cantilevers with a nominal spring constant of $1.6 \mathrm{~N} / \mathrm{m}$, whose tip side was coated with $100-\mathrm{nm}$ thick gold. ${ }^{8}$ The first free resonance frequency $\left(\omega_{1}\right)$ ranged from 25 to $30 \mathrm{kHz}$. In contact-mode AFM, an output voltage of a differential amplifier was kept constant by a feedback controller. On the other hand, in dynamic-mode AFM, a cantilever was vibrated at a fixed angular frequency $\left(\omega_{1}\right)$ by using a piezoelectric actuator. $\omega_{1}$ was set at a signal generator (SG1) to a frequency slightly higher than its first free resonance frequency. The vibration amplitude of the cantilever was then measured utilizing an rms-to-dc converter and its output voltage was kept constant. Extra components required for mapping $\partial C / \partial V$ were another signal generator (SG2), a frequency converter (FC) and an LIA. SG2 was used to supply dc offset voltage $V_{\mathrm{dc}}$ and ac modulation voltage $V_{\text {ac }}$ oscillating at $\omega$. The FC was used to obtain third harmonic frequency $(3 \omega)$ square wave for reference signal of the LIA, which was in-phase to the bias modulation signal. In order to obtain a large $\partial C / \partial V$ signal, $\omega$ (indicated by the black triangles in the spectra in Fig. 2) was chosen so that its third harmonic frequency $(3 \omega)$ came close to the contact resonance frequency $\left(\omega_{c}\right)$ in the case of contact-mode SCFM. On the other hand, $\omega$ was chosen so that $3 \omega$ came

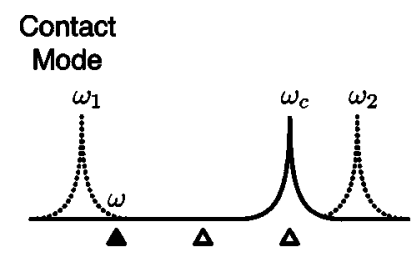

\section{Dynamic
Mode}

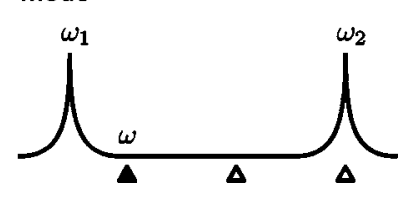

FIG. 2. (a) Schematic of cantilever response spectrum in contact-mode SCFM. The bias modulation frequency $\omega$ (indicated by the black triangle) is chosen so that its third harmonic frequency $(3 \omega)$ comes close to the contact resonance frequency $\left(\omega_{c}\right)$. (b) Schematic of cantilever response spectrum in dynamic-mode SCFM. In this case, $\omega$ (indicated by the black triangle) is chosen so that $3 \omega$ comes close to the second free resonance frequency $\left(\omega_{2}\right)$.

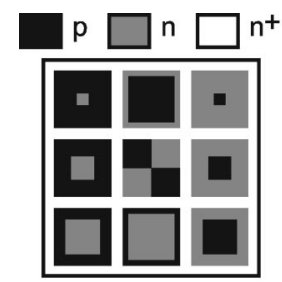

(a)

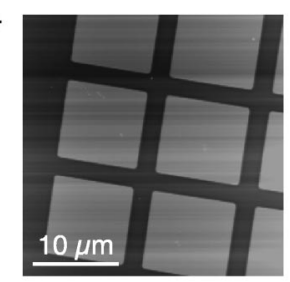

(b)

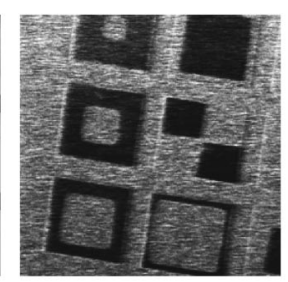

(c)
FIG. 3. (a) Schematic illustration of a unit of doping patterns on Si test sample. Due to a faster oxide growth rate on the $n^{+}$-type region, the $n^{+}$-type region is lower than the other regions by about $50 \mathrm{~nm}$ after oxide etching. (b) $30 \mu \mathrm{m} \times 30 \mu \mathrm{m}$ topographic image of the Si test sample obtained by contact-mode AFM. (c) Contact-mode SCFM ( $\partial C / \partial V)$ image simultaneously obtained with (b). The ac bias modulation amplitude $\left(V_{\mathrm{ac}}\right)$ and the dc bias $\left(V_{\mathrm{dc}}\right)$ applied to the tip were $2 \mathrm{~V} p-p$ and $+2 \mathrm{~V}$, respectively. $p$-type regions are clearly identified as dark regions.

close to the second free resonance frequency $\left(\omega_{2}\right)$ in the case of dynamic-mode SCFM.

We investigated a Si test sample which has differently doped regions. The fabrication process of the test sample was as follows. ${ }^{9}$ A P-doped $n$-type $\mathrm{Si}$ water $(4-6 \Omega \mathrm{cm}, 1$ $\times 10^{15} \mathrm{~cm}^{-3}$ ) was used. $\mathrm{BF}_{2}$ ions were implanted at $70 \mathrm{keV}$ to create $p$-type regions where surface $\mathrm{B}$ density was 2 $\times 10^{16} \mathrm{~cm}^{-3}$. Then As ions were implanted at $120 \mathrm{keV}$ to create $n^{+}$-type regions where the surface As density was 5 $\times 10^{19} \mathrm{~cm}^{-3}$. By a thermal oxidization process and wet etching of oxide by hydrofluoric acid, $n^{+}$regions were made lower than other regions by about $50 \mathrm{~nm}$. Finally, the back side of the wafer was coated with Al by thermal deposition and then the sample was annealed for activation of acceptor and donor ions. Consequently, nine different patterned terraces with $p$-type and $n$-type regions were made on $n^{+}$-type surrounding regions as a unit as shown in a schematic illustration in Fig. 3(a). The size of each terrace was $10 \mu \mathrm{m} \times 10$ $\mu \mathrm{m}$.

At first, SCFM was operated in contact-mode AFM. $\omega_{1}$ and $\omega_{2}$ of the used cantilever were 24.90 and $149.90 \mathrm{kHz}$, respectively. When the tip was made to contact the sample surface at a constant repulsive force of about $5 \mathrm{nN}$, the measured contact resonance frequency $\left(\omega_{c}\right)$ was $107.79 \mathrm{kHz}$. Thus the bias modulation signal from SG2 was set to 35.93 $\mathrm{kHz}, 2 \mathrm{~V}$ peak-to-peak, and the FC generated a $107.79 \mathrm{kHz}$ reference signal, which was supplied to the LIA.

Figures 3(b) and 3(c) are a topographic image of the sample obtained in contact-mode AFM, and an SCFM $(\partial C / \partial V)$ image of the Si test sample, respectively, which were obtained simultaneously. The scanned area was 30 $\mu \mathrm{m} \times 30 \mu \mathrm{m}$. The dc voltage applied to the tip $\left(V_{\mathrm{dc}}\right)$ was +2 $\mathrm{V}$. The time required to obtain these images was $256 \mathrm{~s}$. In the SCFM image, $p$-type regions are clearly identified as dark regions. However, $n$-type and $n^{+}$-type regions are hardly distinguished. It should be noted that the contact resonance frequency $\left(\omega_{c}\right)$ usually varies depending on the contact stiffness on the sample surface. Thus the obtained SCFM image in contact-mode AFM might also contain information on the mechanical properties of the sample, since $\omega$ was chosen so that $3 \omega$ came near to $\omega_{c}$.

Following the contact-mode experiment, SCFM was also operated in dynamic-mode AFM. The cantilever was changed from one used in the contact-mode SCFM experito AIP license or copyright, see http://apl.aip.org/apl/copyright.jsp 


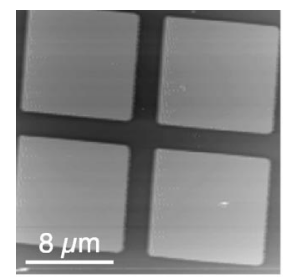

(a)

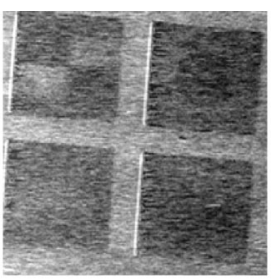

(b)

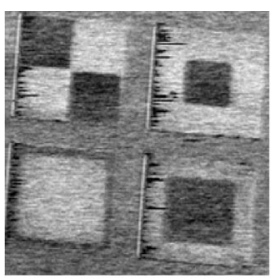

(c)
FIG. 4. (a) $24 \mu \mathrm{m} \times 24 \mu \mathrm{m}$ topographic image of the $\mathrm{Si}$ test sample obtained by dynamic-mode AFM. (b) Dynamic-mode SCFM $(\partial C / \partial V)$ image simultaneously obtained with (a). $V_{\mathrm{ac}}$ and $V_{\mathrm{dc}}$ were $2 \mathrm{~V} p$ - $p$ and $0 \mathrm{~V}$, respectively. (c) Dynamic-mode SCFM image obtained following (b) while $V_{\mathrm{dc}}$ was +2 V. In this case, $p$-type and $n$-type regions are identified as darker and brighter regions compared to $n^{+}$-regions, respectively.

ment. $\omega_{1}$ was $29.50 \mathrm{kHz}$, thus the cantilever was mechanically excited at $29.55 \mathrm{kHz}$. The estimated vibration amplitude was about $20 \mathrm{~nm}$ peak-to-peak. The gap was regulated by keeping the vibration amplitude at about $10 \mathrm{~nm}$ peak-topeak while scanning over the sample surface. Since the second free resonance frequency $\left(\omega_{2}\right)$ was $173.10 \mathrm{kHz}$, the bias modulation signal from SG2 was set to $57.70 \mathrm{kHz}, 2$ V peakto-peak. Then the FC generates $173.10 \mathrm{kHz}$ reference signal.

Figures 4(a) and 4(b) are the topographic image and the dynamic-mode SCFM $(\partial C / \partial V)$ images, respectively, obtained at $V_{\mathrm{dc}}=0 \mathrm{~V}$, which were obtained simultaneously. The scanned area was $24 \mu \mathrm{m} \times 24 \mu \mathrm{m}$. The time required to obtain these images was $256 \mathrm{~s}$. Figure 4(c) is the dynamic SCFM image obtained consecutively at $V_{\mathrm{dc}}=+2 \mathrm{~V}$. In Fig. 4(c), $p$-type regions and $n$-type regions can be identified as darker and brighter regions compared to $n^{+}$regions, respectively. The image contrast in Fig. 4(c) was much clearer compared to that in Fig. 4(b) which was obtained at $V_{\mathrm{dc}}=0$ V. However, the dark features were also prominent at the left-side edges of the $p$ - or $n$-type regions in Fig. 4(c), where the cantilever tip was scanned at the steep terrace edge. These artifacts might be reduced by performing these experiments using a two-path technique, scanning each line first for topography and then retracing the scanned line at a set liftheight for electrical measurement.
Although the ac and dc bias modulation voltages were the same for Figs. 3(c) and 4(c), the obtained image contrast was different. This difference in contrast was due to the fact that the tip-sample distance was different and the fact that both operating modes utilized the resonance enhancement of the cantilever vibration amplitude for the $3 \omega$ component ESF detection.

In summary, by detecting the ESF oscillating at the third harmonic frequency of the bias modulation signal, SCFM $(\partial C / \partial V)$ images were obtained on the Si test sample both in contact-mode and dynamic-mode AFM. We believe that dynamic-mode SCFM would be more promising for improving lateral and capacitance resolution since much higher force sensitivity could be achieved by increasing mechanical $Q$-factor of the cantilever by operating in a vacuum condition.

This work was supported both by a Grand-in-Aid for Scientific Research from the Ministry of Education, Culture, Sports, Science and Technology of Japan and by Industrial Technology Research Grant Program in '01 from New Energy and Industrial Technology Development Organization (NEDO) of Japan. The authors would like to acknowledge the Kyoto University Venture Business Laboratory Project. We would like to thank O. Takai, H. Sugimura (Nagoya Univ.) and N. Nakagiri (Nikon Co.) for kindly providing the Si test sample.

${ }^{1}$ P. De Wolf, R. Stephenson, T. Trenkler, T. Clarysse, T. Hantschel, and W. Vandervorst, J. Vac. Sci. Technol. B 18, 361 (2000).

${ }^{2}$ J. R. Matey and J. Blanc, J. Appl. Phys. 57, 1437 (1985).

${ }^{3}$ R. C. Palmer, E. J. Denlinger, and H. Kawamoto, RCA Rev. 43, 194 (1982).

${ }^{4}$ C. C. Williams, W. P. Hough, and S. A. Rishton, Appl. Phys. Lett. 55, 203 (1989).

${ }^{5}$ R. C. Barrett and C. F. Quate, J. Appl. Phys. 70, 2725 (1991).

${ }^{6}$ Y. Martin, D. W. Abraham, and H. K. Wickramasinghe, Appl. Phys. Lett. 52, 1103 (1988).

${ }^{7}$ D. W. Abraham, C. Williams, J. Slinkman, and H. K. Wickramasinghe, J. Vac. Sci. Technol. B 9, 703 (1991).

${ }^{8}$ After the submission of the original manuscript, we succeeded in obtaining similar or even better results by using highly-doped uncoated tip.

${ }^{9}$ H. Sugimura, Y. Ishida, K. Hayashi, O. Takai, and N. Nakagiri, Appl. Phys. Lett. 80, 1459 (2002). 